\title{
Aldosterone Modulates Blood Homocysteine and Cholesterol in Coronary Artery Disease Patients - a Possible Impact on Atherothrombosis?
}

\author{
K. KAROLCZAK ${ }^{1}$, P. KUBALCZYK ${ }^{2}$, R. GLOWACKI ${ }^{2}$, R. PIETRUSZYNSKI ${ }^{3}$, C. WATALA $^{1}$ \\ ${ }^{1}$ Department of Hemostatic Disorders, Medical University of Lodz, Lodz, Poland, ${ }^{2}$ Department of \\ Environmental Chemistry, Faculty of Chemistry, University of Lodz, Lodz, Poland, ${ }^{3}$ Department of \\ Radiological and Nuclear Diagnostics and Therapy, Veterans' Central Hospital, Lodz, Poland
}

Received May 20, 2017

Accepted October 30, 2017

On-line January 5, 2018

\section{Summary}

Aldosterone plays a key role in maintaining the homeostasis of the whole organism. Under some circumstances, aldosterone can contribute to the progression of cardiovascular diseases, including coronary artery disease. This study demonstrates that aldosterone associates negatively with some lipidogram parameters and positively with the concentration of homocysteine. These associations are characteristic for coronary artery disease and are not present in control subjects. The findings also indicate that in vitro aldosterone stimulates homocysteine production by rat adrenal glands, which may explain the associations observed with coronary artery disease. Moreover, we have found that aldosterone significantly modulates in vitro platelet reactivity to arachidonate and collagen - aldosterone increases the pro-aggregatory action of collagen, but decreases the pro-aggregatory potential of arachidonate. Therefore, the findings of these in vitro and ex vivo experiments indicate the existence of new pathways by which aldosterone modulates lipid- homocysteine- and platelet-dependent atherogenesis.

\section{Key words}

Aldosterone - Atherosclerosis • Metabolism - Cardiovascular risk factors $\bullet$ Homocysteine $\bullet$ Cholesterol $\bullet$ Platelet aggregation

\section{Corresponding author}

K. Karolczak, Department of Hemostatic Disorders, Chair of Biomedical Sciences, Medical University of Lodz, ul. Mazowiecka 6/8, 92-215 Lodz, Poland. E-mail: kamilkarolczak@gmail.com or kamil.karolczak@umed.lodz.pl

\section{Introduction}

Aldosterone, due to its regulatory action on both the ion balance and the volume of body fluids (Booth et al. 2002), plays a key role in maintaining the homeostasis of the whole organism. However, under some circumstances, it may exert harmful effects, leading to the progression of cardiovascular diseases, including coronary artery disease (CAD). The role of aldosterone in atherosclerosis has been well documented in clinical studies, showing a positive association between plasma aldosterone level and the risk of mortality and the occurrence of major vascular events (Hillaert et al. 2013, Ivanes et al. 2013). Moreover, plasma aldosterone concentration may predict the risk of in-stent restenosis in patients after angioplasty (Amanao et al. 2006). Little is known of whether aldosterone can influence the progression of atherosclerosis through the modulation of the metabolism of lipids, carbohydrates or low-molecular-weight thiols (LMWT), like homocysteine (Hcy), cysteine (Cys), cysteinylglycine (CysGlyc) and glutathione (GSH), however this is plausible, since Musani et al. (2013) report that plasma aldosterone associates with various components of metabolic syndrome.

The potential impact of aldosterone on blood platelet-dependent pathogenic changes in hemostasis has not been widely explored. A previous report has indicated that aldosterone does not modulate ADP-dependent activation of blood platelets, and so aldosterone has no 
hemostatic properties (Moraes et al. 2005). Interestingly, the presence of the mineralocortioid receptor subunits, occurring as heterodimers of mineralo- and glucocorticoid receptor subunits in the platelet membrane supports the hypothesis that platelets should respond to aldosterone stimulation. The present study monitors how aldosterone modulates the whole blood platelet response to either arachidonic acid or collagen, the agonists different than those used by Moraes et al. (2005). Accordingly, we hypothesize that platelet irresponsiveness or responsiveness to aldosterone treatment is not a generalized phenomenon, but individually depends upon the used platelet agonist.

Among "classical" atherogenic risk factors, cholesterol fractions and triglycerides have been the best characterized with regard to their relationship with plasma aldosteronemia. In addition, one of the newly recognized players in hemostasis, Hcy (Karolczak and Olas 2009), has not yet been evaluated as a compound potentially associated with aldosterone. The aim of the present study was to re-evaluate known atherothrombosis risk factors (lipids) and to evaluate newly-reported ones (LMWT) in the context of their associations with concentrations of aldosterone.

\section{Methods}

\section{Chemicals}

Arachidonic acid and equine tendon collagen type I for platelet aggregation were obtained from Chrono-Log Corp. (Havertown, PA, USA). Sterile physiological saline $(0.9 \% \mathrm{NaCl})$ from Polpharma (Starogard Gdanski, Poland) and sterile PBS from Biomed (Krakow, Poland). EDTA and serum probes for blood sampling were provided by Sarstedt AG \& Co. (Numbrecht, Germany). Ketamine and sedazine were purchased from Biowet-Pulawy (Poland). Chromatographic columns were from Agilent Technologies, Inc. (Santa Clara, CA, USA). Aldosterone, eplerenone, dimethyl sulfoxide (DMSO), sodium hydrogen phosphate heptahydrate $\left(\mathrm{Na}_{2} \mathrm{HPO}_{4} \cdot 7 \mathrm{H}_{2} \mathrm{O}\right)$, sodium dihydrogen phosphate, tris(2-carboxyethyl) phosphine, perchloric acid, acetonitryl, trichloroacetic acid and sodium hydroxide and Dulbecco's Modified Eagle's Medium were from Sigma-Aldrich (St. Louis, MO, USA). 2-chloro-1-methylquinolinium tetrafluoroborate (CMQT) was prepared in this laboratory according to the procedure described earlier (Bald and Głowacki 2001).

\section{Subjects}

Blood donors were enrolled to the project from a group of patients visiting a specialist cardiology clinic. Enrolment was conducted in accordance with the Declaration of Helsinki (2008) of the World Medical Association. All steps of volunteer recruitment were accepted by the Local Ethical Committee. Each of the volunteers gave their informed consent before the beginning of experimental procedures.

54 control subjects not suffering from CAD and 59 patients diagnosed with $\mathrm{CAD}$ were enrolled to the study.

A diagnosis of coronary artery disease was made on the base of echocardiography or/and angiography, in which at least one coronary vessel was observed to be occluded by at least $50 \%$.

The exclusion criteria were as follows: diagnosed unstable angina pectoris, chronic renal failure, type 1 diabetes mellitus, allergic diseases, autoimmune diseases, infectious diseases, acute infection in the previous two weeks, acute coronary syndrome within six months before the experiment, any type of inflammatory disease or non-chronic inflammation or malignancies. Alcohol abusers, drug abusers, those who used alcohol on the day before the experiment, those suffering from psychiatric disease or any other chronic diseases other than $\mathrm{CAD}$, those using some specific diets or taking diet supplements, subjects with unclear clinical status and pregnant women were also excluded from the study.

For each participant, the following variables were recorded: age, sex, height, weight, hip and waist dimensions, current and past smoking status, stress exposure, physical and professional activity, history of previous myocardial infarction, ischemic brain stroke or transient ischemic attack, percutaneous coronary intervention (PCI), coronary artery bypass grafting (CABG), other concomitant diseases or current pharmacotherpies (including antihypertnesive, hypolipidemic and antidiabetic drugs).

\section{Measurements of blood morphology and biochemistry}

EDTA-anticoagulated plasma samples were used in all ELISA and HPLC measurements. Blood samples were taken from subjects between 8:00-10:00 AM following 12-h fasting. Blood plasma was separated from the whole blood immediately after sampling $(1000 \mathrm{x} \mathrm{g} /$ $15 \mathrm{~min} / 4^{\circ} \mathrm{C}$ ). Plasma aliquots were stored at $-70{ }^{\circ} \mathrm{C}$ and thawed only once prior to testing.

Concentrations of aldosterone and cystatin $\mathrm{C}$ were measured with commercially-available ELISA kits 
(DRG Instruments GmbH, Marburg, Germany), according to the manufacturer's instructions provided with the kits.

Standard measurements of blood morphology parameters and blood serum biochemistry were performed with a 5-Diff Sysmex XS-1000i (Sysmex, Kobe, Japan) and an DIRUI CS 400 analyzer (Dirui, Changchun, China).

\section{HPLC measurements of low-molecular-weight thiol concentrations}

The $50 \mu \mathrm{l}$ aliquots of plasma or DMEM, taken after the incubations of adrenal glands with aldosterone, were diluted twice with $0.2 \mathrm{~mol} / \mathrm{l}$ phosphate buffer (pH 7.6) and supplemented with $10 \mu \mathrm{l}$ of $0.25 \mathrm{~mol} / \mathrm{l}$ solution of tris(2-carboxyethyl)phosphine phosphate buffer. The mixture was vortexed and left for $10 \mathrm{~min}$, supplemented with $5 \mu \mathrm{l}$ of $0.1 \mathrm{~mol} / \mathrm{l}$ solution of 2-chloro1-methylquinolinium tetrafluoroborate and left for $2 \mathrm{~min}$. Next, $25 \mu \mathrm{l}$ of $3 \mathrm{~mol} / \mathrm{l}$ perchloric acid was added and the precipitated protein was pelleted by centrifugation (10 $\mathrm{min} / 12,000 \times \mathrm{g})$. The supernatant was then transferred into a separate vial and the $20 \mu \mathrm{l}$ portion was injected onto the $150 \times 4.6 \mathrm{~mm}$ ZORBAX SB C18 column packed with $5 \mu \mathrm{m}$ particles. The mobile phase (flow rate: $1 \mathrm{ml} / \mathrm{min}$; temperature: $25^{\circ} \mathrm{C}$ ) consisted of $0.1 \mathrm{~mol} / 1$ trichloroacetic acid (solution $\mathrm{A}$ ), adjusted to $\mathrm{pH} 1.65$ with a $1 \mathrm{~mol} / 1$ sodium hydroxide solution, and acetonitrile (solution $\mathrm{B}$ ). The elution profile was as follows: 0-8 min, 11-40\% $\mathrm{B}$; 8-12 min, $40-11 \% \mathrm{~B}$; (A/B, v/v). The identification of peaks was based on the comparison of retention times and diode-array spectra with a corresponding set of data obtained by the analysis of authentic compounds. Detection and quantification were conducted by UV absorbance at $355 \mathrm{~nm}$.

\section{Isolation and incubation of animal tissue samples}

All experiments with animals were approved by the Local Committee on Standardization and Ethics for Experiments with Animals.

Male Sprague-Dawley rats weighing 250-300 g were used. They were bred in groups of five animals per one cage. The rats received standard Murigran laboratory chow (Motycz, Poland) and water ad libitum, and a light : darkness $/ 12 \mathrm{~h}: 12 \mathrm{~h}$ cycle was maintained in the culture room for the whole period of the experiment.

The animals were deeply anesthetized with an i.m. injection of ketamine. $\mathrm{HCl}(100 \mathrm{mg} / \mathrm{kg})$ and sedazine $(10 \mathrm{mg} / \mathrm{kg})$. When deep anesthesia was achieved, the skin and muscles were cut in the midline of the abdomen and the abdominal cavity was opened. The aorta was carefully dissected and catheterized with a Venacat-G24 venous cannula (Polfa, Boleslawiec, Poland). Blood was collected from the abdominal aorta of the anesthetized rats and used in other experiments. After exsanguination, both adrenal glands were rapidly excised from each animal and placed in Dulbecco's Modified Eagle's Medium (DMEM) for $20 \mathrm{~min}$ at room temperature. After this period, the adrenal glands were dissected from the fatty remains, decapsulated and cut into small pieces. The combined fragments of the left and right adrenal glands (randomized in blocks) were weighed (to obtain the wet weight of the glands) and placed in two wells filled with DMEM medium supplemented with a cocktail of antibiotics containing $10 \mu \mathrm{g} / \mathrm{ml}$ gentamicin and $0.25 \mu \mathrm{g} / \mathrm{ml}$ amphotericin $\mathrm{B}$, and protease inhibitors (Thermo Fisher Scientific, Waltham, MA, USA). Aldosterone or its vehicle (DMSO, final conc. $0.00001 \%$ ) was added to different wells at final concentrations of 50 or $500 \mathrm{pg} / \mathrm{ml}$. Controls were also created with the same concentrations of the vehicle as in the aldosterone-treated samples. Adrenal glands were incubated with aldosterone for up to $12 \mathrm{~h}$ at $37^{\circ} \mathrm{C}$ $\left(\mathrm{O}_{2} / \mathrm{CO}_{2}=95 / 5 \%\right)$. Following the incubation, the $100 \mu \mathrm{l}$ aliquots of culture media from the wells with tissues were carefully removed, immediately placed in Eppendorf tubes and frozen at $-70{ }^{\circ} \mathrm{C}$ for storage until use.

\section{Impedance aggregometry of human blood platelets}

For experiments testing impact of aldosterone on platelet aggregation in vitro we used blood obtained from healthy donors (males/females $=6 / 5$ ) at mean age $34.5 \pm 10.8$ years. All recruited volunteers declared that they have not been taking any antiplatelet drugs, including acetylsalicylic acid, neither complex drugs including acetylsalicylic acid nor any antiinflammatory and analgesic medications at least for 14 days preceding blood sampling. All donors were non-smokers and apparently healthy subjects. Blood was taken from peripheral vein, with an 18-gauge needle into a tubes containing buffered sodium citrate at concentration of $0.105 \mathrm{~mol} / \mathrm{l}$ (citrate/blood ratio $=1 / 9, \mathrm{vol} / \mathrm{vol})$. All steps of experiments provided with healthy volunteers were previously accepted by the Bioethical Committee at the Medical University in Lodz (Poland).

Aggregometric measurements were performed in whole blood using a Multiple Platelet Function Analyzer (Dynabyte Medical, Munchen, Germany). Aliquots of $350 \mu$ of whole blood were incubated with aldosterone 
at final concentrations of 50,150 or $500 \mathrm{pg} / \mathrm{ml}(15 \mathrm{~min}$, RT). In some experiments, blood incubation with aldosterone was preceded by $15 \mathrm{~min}$ incubation with eplerenone $(50 \mu \mathrm{mol} / \mathrm{l})$. All the incubations were carried out at room temperature. Following the incubations, blood samples were supplemented with either $0.5 \mathrm{mmol} / 1$ arachidonic acid or $1 \mu \mathrm{g} / \mathrm{ml}$ collagen, and whole blood platelet aggregation was monitored for a further $15 \mathrm{~min}$ $\left(37^{\circ} \mathrm{C}\right)$. Two parameters describing platelet aggregation were recorded: the area under the aggregation curve (AUC) and the maximal aggregation $\left(\mathrm{A}_{\max }\right)$. These were used to calculate the extent of platelet responsiveness to agonists according to the formula: (AUC x $\left.\mathrm{A}_{\max }\right) / 1000$.

\section{Statistical analysis}

Outliers, the normality of data distribution and homogeneity of variances were verified respectively with Grubbs' test, the Shapiro-Wilk test and Levene's test. Depending on the normality of distribution, data was presented as mean $\pm \mathrm{SD}$ or median and interquartile range (IQR: lower quartile, LQ [25\%], to upper quartile, UQ [75\%]). The unpaired Student's t-test, Mann-Whitney U-test or one-way models of ANOVA were employed as simple inference tests. Analysis of covariance (ANCOVA) was used to evaluate the impact of selected accompanying collinear variables in the inference testing on the corrected measures of central tendency and to calculate the adjusted measures (means). For multivariate analyses (multiple regression, partial and semipartial correlation, covariance analysis), the Box-Cox-transformed raw data was used to ensure normal distribution of residuals, homoscedasticity of variables and regression slope homogeneity. In addition, due to relatively small sample sizes and the low statistical power of some estimated inferences in some calculations, the resampling bootstrap technique (10,000 iterations) was used to determine how likely it would be to obtain the revealed differences due to pure chance. In such circumstances, the bootstrap-engineered test statistics (1,000 iterations) are referred to instead of the classical ones. Statistical analysis was performed using Statistica v. 12.5 and Resampling Stats Add-in for Excel v. 4.

\section{Results}

Clinical and biochemical characteristics of the investigated CAD and non-CAD patients

The demographic, anthropometric, selected medical and biochemical variables recorded in non-CAD and CAD patients are presented in Table 1.

CAD patients were significantly older and characterized by higher $\mathrm{BMI}$ in comparison to non-CAD volunteers.

Analysis of blood morphological parameters revealed few significant differences between non-CAD and CAD-affected subjects. Patients suffering from CAD had higher total white blood cell counts (WBC) and the numbers of lymphocytes, neutrophils and monocytes remained elevated (data not shown).

The patients with CAD had insignificantly greater plasma creatinine and CRP and significantly higher fasting glucose. The difference in fasting glycemia remained significant upon the exclusion of patients diagnosed with type 2 diabetes. Higher triglyceride levels were noted in the CAD group. As the majority of CAD patients $(76.3 \%$ in $\mathrm{CAD}$ vs. $26.4 \%$ in non-CAD patients) were on hypolipidemic treatment (HMG-CoA reductase inhibitors, statins), total cholesterol and its lipoprotein subfractions remained significantly lower in the CAD group compared to non-CAD individuals. Patients with CAD were characterized by higher blood plasma homocysteinemia than non-CAD subjects (Table 1).

It was then examined whether non-CAD and CAD groups could be significantly differentiated by Hcy concentration after homocysteinemia values were corrected for age and creatinine level, i.e. variables which were strongly associated with homocysteinemia, (ANCOVA). After such a correction, Hcy concentration was still significantly higher in the CAD group $(P<0.001$ in the one-sided Student's t-test).

Plasma aldosterone concentrations were almost equal in both tested groups (Median; IQR: 58.5; $35.3-80.2 \mathrm{pg} / \mathrm{ml}$ in non-CAD patients vs. 57.0; 39.7-74.2 pg/ml in CAD subjects; n.s. by Mann-Whitney U-test). Interestingly, correcting the plasma aldosteronemia values for age and fasting glycemia (ANCOVA) revealed that CAD patients have significantly higher aldosterone plasma levels than non-CAD subjects $(P<0.001$ in one-sided Student's t-test). Significant positive correlations were found between Hcy and aldosterone level in both the CAD patient group $\left(R_{\mathrm{S}}=0.287, P<0.00001\right)$ and non-CAD control group $\left(R_{\mathrm{S}}=0.232, P<0.001\right)$ after Hcy correction for age and creatinine, and aldosterone correction for age and glycemia-level. 
Table 1. Demographic, clinical, social and biochemical characteristics of investigated populations of patients.

\begin{tabular}{|c|c|c|}
\hline & non-CAD & CAD \\
\hline \multicolumn{3}{|l|}{ Demographic } \\
\hline Age (years) & $60.4 \pm 9.4$ & $64.8 \pm 10.8^{\mathrm{a} \#}$ \\
\hline Sex (male/female) & $33 / 21$ & $44 / 15$ \\
\hline \multicolumn{3}{|l|}{ Clinical } \\
\hline$B M I\left(\mathrm{~kg} / \mathrm{m}^{2}\right)$ & $27.2 \pm 3.5$ & $29.4 \pm 4.5$ \\
\hline Diabetes mellitus (type 2) (\%) & 13.0 & 32.2 \\
\hline WHR & $0.9 \pm 0.2$ & $0.9 \pm 0.1$ \\
\hline Previous MI (\%) & 0 & 56 \\
\hline$P C I(\%)$ & 0 & 69.5 \\
\hline$C A B G(\%)$ & 0 & 16.9 \\
\hline Previous stroke or TIA (\%) & 1.9 & 13.5 \\
\hline \multicolumn{3}{|l|}{ Social and lifestyle } \\
\hline \multicolumn{3}{|l|}{ Smoking } \\
\hline Current (\%) & 5.6 & 6.7 \\
\hline In the past (\%) & 86.8 & 19 \\
\hline \multicolumn{3}{|l|}{ Medication } \\
\hline \multicolumn{3}{|l|}{ Antiplatelet drugs } \\
\hline Acetylsalicylic acid (\%) & 18.5 & 59.3 \\
\hline Purinergic receptor antagonists (\%) & 0 & 32.3 \\
\hline \multicolumn{3}{|l|}{ Hypolipidemic drugs } \\
\hline Statins (\%) & 25.9 & 83.0 \\
\hline Fibrates (\%) & 0 & 3.4 \\
\hline \multicolumn{3}{|l|}{ Antihypertensive drugs } \\
\hline ACE-inhibitors (\%) & 44.4 & 64.4 \\
\hline$\beta$-blockers $(\%)$ & 10.2 & 79.6 \\
\hline Ca-channel blockers (\%) & 2.2 & 1.7 \\
\hline \multicolumn{3}{|l|}{ Hypoglycemic drugs } \\
\hline Insulin $(\%)$ & 5.5 & 20.3 \\
\hline Sulfonylureas (\%) & 9.2 & 3.4 \\
\hline Metformin (\%) & 3.7 & 11.9 \\
\hline \multicolumn{3}{|l|}{ Blood morphology } \\
\hline$W B C\left(\times 10^{3} / \mu l\right)$ & $6.1 \pm 1.8$ & $6.9 \pm 1.6^{\mathrm{b}^{*}}$ \\
\hline$N E U\left(\times 10^{3} / \mu l\right)$ & $3.6 \pm 1.2$ & $4.1 \pm 1.3^{\mathrm{a}^{*}}$ \\
\hline $\operatorname{LYM}\left(\times 10^{3} / \mu l\right)$ & $1.7 \pm 0.6$ & $1.9 \pm 5.1^{\mathrm{a}^{*}}$ \\
\hline $\operatorname{MON}\left(\times 10^{3} / \mu l\right)$ & $0.4 \pm 0.1$ & $0.45 \pm 0.1^{\mathrm{a}^{*}}$ \\
\hline \multicolumn{3}{|l|}{ Selected blood plasma metabolites } \\
\hline Creatinine $(\mu \mathrm{mol} / \mathrm{l})$ & $94.2 \pm 15.7$ & $97.2 \pm 16.8$ \\
\hline Total cholesterol $(\mathrm{mmol} / \mathrm{l})$ & $5.4 \pm 1.3$ & $4.8 \pm 1.2^{\mathrm{c}^{*}}$ \\
\hline HDL cholesterol ( $\mathrm{mmol} / \mathrm{l})$ & $1.4 \pm 0.3$ & $1.3 \pm 0.3^{\mathrm{a}^{*}}$ \\
\hline LDL cholesterol ( $\mathrm{mmol} / \mathrm{l})$ & $3.7 \pm 1.1$ & $3.1 \pm 1.0^{\mathrm{b}^{*}}$ \\
\hline Triglycerides (mmol/l) & $1.4 \pm 0.6$ & $1.6 \pm 0.6$ \\
\hline Glucose $(\mathrm{mmol} / \mathrm{l})^{+}$ & $6.6 \pm 1.6$ & $8.3 \pm 3.3^{\mathrm{c}^{*}}$ \\
\hline Glucose $(\mathrm{mmol} / \mathrm{l})^{\dagger+}$ & $6.3 \pm 1.0$ & $6.9 \pm 1.7^{\mathrm{a}^{*}}$ \\
\hline Homocysteine $(\mu \mathrm{mol} / \mathrm{l})$ & $7.4 \pm 1.9$ & $9.0 \pm 3.4^{* b}$ \\
\hline Glutathione $(\mu \mathrm{mol} / \mathrm{l})$ & $4.9 \pm 1.3$ & $5.0 \pm 1.2$ \\
\hline Cysteine $(\mu \mathrm{mol} / \mathrm{l})$ & $159.5 \pm 23.9$ & $167.5 \pm 29.3$ \\
\hline Cysteinylglycine ( & $26.4 \pm 5.0$ & $25.7 \pm 5.2$ \\
\hline
\end{tabular}

Variables expressed as means \pm SD or percentage fractions of whole groups of investigated patients ( $n=53$ non-CAD and $n=59$ for CAD patients). Comparisons between non-CAD and CAD groups performed with unpaired Student's t-test $\left(^{\#}\right)$ or Mann-Whitney U-test (*). Comparisons of glucose concentrations between non-CAD and CAD patients were done in subgroups including $\left({ }^{\dagger}\right)$ or excluding $\left({ }^{+\dagger}\right)$ patients with diabetes. ${ }^{\mathrm{a}} P<0.05,{ }^{\mathrm{b}} P<0.005,{ }^{\mathrm{c}} P<0.001$. BMI, Body Mass Index; CABG, Coronary Artery Bypass Grafting surgery; CAD, Coronary Artery Disease; LYM, Lymphocytes; MI, Myocardial Infarction; MON, Monocytes; NEU, Neutrophiles; PCI, Percutaneous Coronary Intervention; TIA, Transient Ischemic Attack; WBC, White Blood Cell count; WHR, Waist-Hip Ratio. 


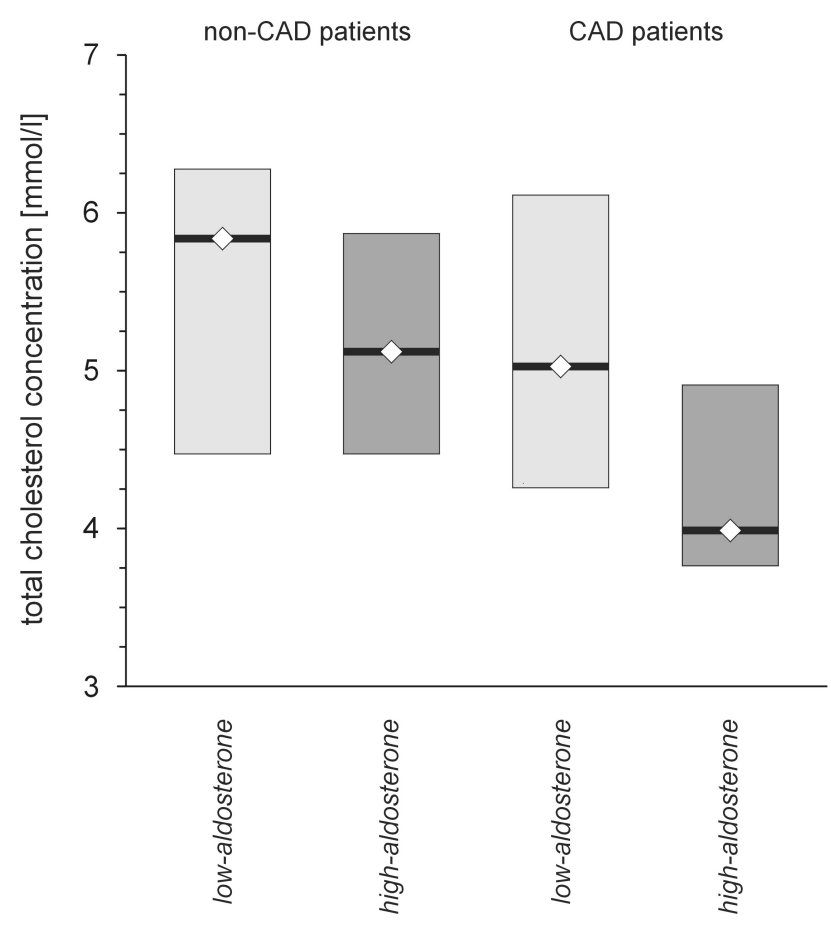

Fig. 1. Blood total cholesterol in non-CAD and $C A D$ patients differentiated according to plasma aldosterone. Data presented as median values (solid horizontal line) with IQR (vertical rectangles) in non-CAD subjects with lower (light grey boxes, $n=27$ ) or higher (dark grey boxes, $n=27$ ) aldosterone concentrations, and in CAD-affected patients with lower (light grey boxes, $n=30$ ) or higher (dark grey boxes, $n=29$ ) aldosterone levels. In all groups, the values of cholesterol concentrations are corrected for the use of statins (ANCOVA on the Box-Cox-transformed data). Significance of differences verified with two-way ANOVA on the Box-Cox-transformed data: $P<0.005$ for the effect of CAD, $P<0.05$ for the effect of low-high aldosterone. For details of statistical comparisons see Results.

Comparison of lipidogram parameters in control subjects and CAD patients with lower and higher aldosterone plasma levels

Using the median values of plasma aldosterone levels in non-CAD and CAD patients, the examined populations of non-CAD and $\mathrm{CAD}$ individuals were divided into two subgroups: one showing plasma aldosterone levels equal or lower than the median and the other with the aldosterone concentration higher than the median value.

In non-CAD subgroups, no significant differences were observed for lipidogram parameters, regardless of plasma aldosterone level. No association was found between plasma aldosteronemia and lipid parameters in the control non-CAD group. Otherwise, in CAD subjects with lower plasma aldosterone levels (Median; IQR: 39.7; 31.0-50.6 pg/ml), significantly elevated total- (by $21 \%$ ) and LDL-cholesterol (by $23 \%$ ) were found compared to the CAD individuals with higher plasma aldosterone concentrations (Median; IQR: 75.3; $65.0-104.0 \mathrm{pg} / \mathrm{ml} ; \quad P<0.001$ by Mann-Whitney U-test) (by the non-paired Student's t-test for total- and LDL-cholesterol) (Fig. 1).

The median values of HDL-cholesterol did not differ significantly between low-aldosteronic and highaldosteronic CAD patients.

When the groups were divided according to aldosterone plasma level, they were found to have equal median triglyceride concentrations (Median: $1.5 \mathrm{mmol} / \mathrm{l}$ for both groups, IQR: 1.2-2.1 and 1.1-1.9 for lower and higher aldosterone groups, respectively), and no significant difference was found for triglyceride parameters.

The correlation analysis demonstrated statistically significant inverse correlations between aldosteronemia level and total- $\left(R_{\mathrm{S}}=-0.347 ; P<0.001\right)$ and LDL-cholesterol $\left(R_{\mathrm{S}}=-0.317 ; P=0.01\right)$ levels in the total CAD group. However, no significant correlations were revealed between aldosterone level and HDL-cholesterol or triglyceride concentrations.

Blood plasma homocysteinemia in control subjects and CAD patients stratified according to creatinine and aldosterone plasma concentrations

The effects of plasma aldosterone differed considerably between $\mathrm{CAD}$ and non-CAD patients $(P<0.01$ by two-way ANOVA). In the population of patients not suffering from $\mathrm{CAD}$, stratified according to plasma aldosterone levels, the concentrations of plasma Hcy were equal in both non-CAD subgroups (Fig. 2). Plasma aldosteronemia and homocysteinemia revealed no significant correlations among non-CAD patients.

In patients suffering from $\mathrm{CAD}$, the plasma levels of homocysteine remained higher in those who exhibited higher plasma aldosterone concentrations than those with lower aldosteronemia $(P<0.04$ by two-way ANOVA and the post hoc multiple comparisons LSD Fisher's test on the Box-Cox transformed data) (Fig. 2). In CAD individuals, a significant positive correlation was found between plasma homocysteinemia and aldosteronemia $\left(R_{\mathrm{S}}=0.392, P<0.01\right)$.

In the control group, plasma homocysteinemia was positively associated with plasma creatinine level $\left(R_{\mathrm{S}}=0.547, \quad P<0.001\right)$. Creatinine also positively correlated with the plasma concentrations of other thiols: cysteine $\left(R_{\mathrm{S}}=0.271, \quad P<0.05\right) \quad$ and cysteinylglycine $\left(R_{\mathrm{S}}=0.526, P<0.001\right)$. In CAD-affected patients, plasma Hcy concentrations positively correlated with plasma 
creatinine levels $\left(R_{\mathrm{S}}=0.374, P<0.01\right)$. Of other thiols tested in CAD patients, only cysteine was found to be positively associated with creatinine $\left(R_{\mathrm{S}}=0.413\right.$, $P=0.001)$. Plasma creatinine level was also positively correlated with plasma aldosteronemia $\left(R_{\mathrm{S}}=0.270\right.$, $P<0.05)$.

In both subgroups plasma creatinine positively associated with plasma concentrations of cystatin $\mathrm{C}$ ( $R_{\mathrm{S}}=0.356, P<0.01$ in non-CAD group and $R_{\mathrm{S}}=0.266$, $P<0.05$ in CAD group). On the other hand, cystatin $\mathrm{C}$ did not reveal any significant correlations with the investigated biothiols or aldosterone, in either of the studied groups (data not shown).

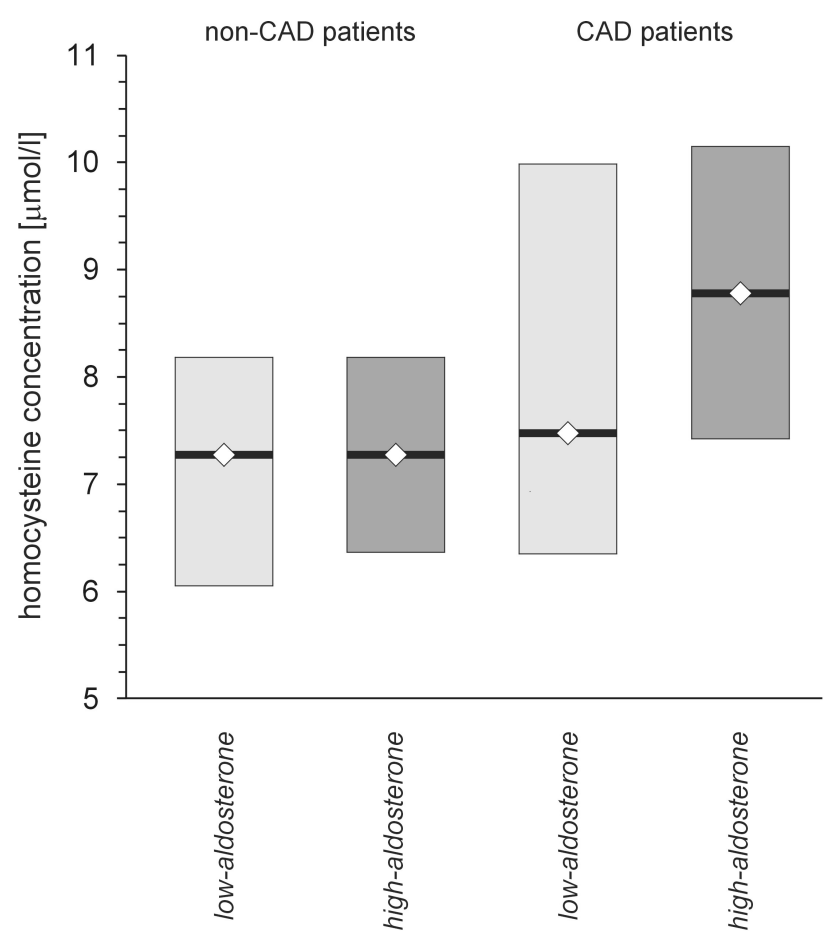

Fig. 2. Homocysteine in peripheral blood plasma in non-CAD and CAD patients differentiated according to plasma aldosterone levels. Data presented as median values (solid horizontal line) with IQR (vertical rectangles) in non-CAD subjects with lower (light grey boxes, $n=27$ ) or higher (dark grey boxes, $n=27$ ) aldosterone concentrations, and in CAD-affected patients with lower (light grey boxes, $n=30$ ) or higher (dark grey boxes, $n=29$ ) aldosterone levels. Significance of differences verified with two-way ANOVA on the Box-Cox-transformed data: $P<0.01$ for the effect of CAD, n.s. for the effect of low-high aldosterone. For details of statistical comparisons see Results.

In vitro effect of aldosterone on the release of Hcy from rat adrenal glands

As our findings revealed an association between aldosterone and Hcy, the next step was to use an in vitro model to verify the possible aldosterone-dependent stimulation of Hcy excretion from the main source of this mineralocroticoid, i.e. the adrenal glands. Following a long, 12-h incubation of adrenal glands with aldosterone at its final concentration of $500 \mathrm{pg} / \mathrm{ml}$, a marked increase in Hcy level (by $84 \%$ ) was observed (Fig. 3).

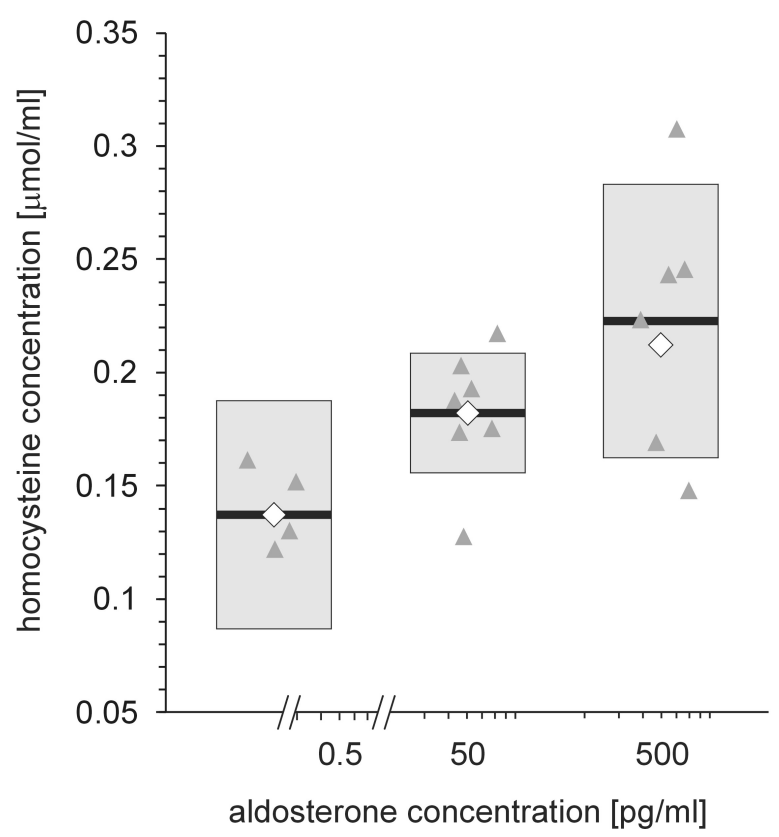

Fig. 3. Homocysteine release from rat adrenal glands incubated in vitro with aldosterone. Data presented as mean (solid horizontal line) $\pm 95 \%$ CI (vertical rectangles) and standardized per one milligram of adrenal gland wet weight, $n=3-7$; the raw data for the increasing concentrations of aldosterone marked as solid triangles. Statistical significance of linear trend estimated with one-way ANOVA; $P_{\text {bootstrap }}<0.003$. For further details of statistical comparisons see Results.

No such effect was observed for shorter incubation times (not shown). In addition, neither shorter nor longer incubation of adrenal glands with lower aldosterone concentrations changed the Hcy level in the collected incubation media compared to tissue samples incubated with the aldosterone vehicle (0.00001\% DMSO) (by two-way ANOVA).

The use of corticosterone ( 100 and $500 \mathrm{pg} / \mathrm{ml}$ ) or ACTH (100 and $600 \mathrm{pg} / \mathrm{ml})$ as potential modulators of the release of Hcy from rat adrenal glands in similar in vitro models was not associated with any significant changes in Hcy levels in the incubation media (data not shown).

In addition to Hcy concentration, we also investigated the fluctuations in the concentrations of reduced glutathione (GSH) and cysteine (Cys) in culture media used for the incubation of dissected rat adrenal 
glands after their incubation with all the tested hormones. Concentrations of both GSH and Cys in the culture media harvested after the incubation of adrenal glands with aldosterone, corticosterone and $\mathrm{ACTH}$ were not significantly different than those noted in control samples $(P<0.05)$, regardless of the used hormone concentrations and incubation times (data not shown).

Impact of aldosterone on collagen- and arachidonic acid-dependent aggregation

Both collagen $(1 \mu \mathrm{g} / \mathrm{ml})$ and arachidonate $(0.5 \mathrm{mmol} / \mathrm{l})$ stimulated the whole blood platelet aggregation to $469.7 \pm 232.6$ [mean $\pm \mathrm{SD}$; a.u.] and 413.6 (404.1; 426.7) [median (IQR); a.u.]. When blood samples were treated with increasing aldosterone concentrations $(50,150$ or $500 \mathrm{pg} / \mathrm{ml})$ the collagen-induced platelet aggregation increased by $38.7 \pm 32.7 \%, 42.5 \pm 74.2 \%$ and $33.2 \pm 19.3 \%$, respectively for 50,150 and $500 \mathrm{pg} / \mathrm{ml}$, with respect to baseline aggregation values; however, these elevations were not statistically significant. The prior treatment of blood samples with eplerenone reduced the aldosterone-augmented collagen-induced platelet aggregation (by $47.0 \pm 34.9 \%, P<0.01$, by $53.0 \pm 20.2 \%$, $P<0.0002$ and by $42.6 \pm 51.5 \%, P<0.02$, respectively for 50,150 and $500 \mathrm{pg} / \mathrm{ml}$, with respect to aldosterone alone). In the presence of aldosterone, the extent of the arachidonate-induced aggregation decreased by $21.3 .7 \pm 11.7 \%$ for $150 \mathrm{pg} / \mathrm{ml} \quad(P<0.02)$, and by $19.4 \pm 19.6 \%$ for $500 \mathrm{pg} / \mathrm{ml}(P=0.065)$, while eplerenone further suppressed the aldosterone-reduced arachidonateinduced platelet aggregation by $34.5 \pm 26.1 \%$ for $50 \mathrm{pg} / \mathrm{ml}, \quad(P<0.04)$, by $32.7 \pm 37.5 \%$ for $150 \mathrm{pg} / \mathrm{ml}$, $(P=0.057)$ and by $38.7 \pm 33.2 \%$ for $500 \mathrm{pg} / \mathrm{ml},(P<0.04)$, with respect to aldosterone alone; in all the inference analyses, the bootstrap-boosted approaches of the ANOVA with repeated measures and paired Student's t-test with the Bonferroni's correction for multiple comparisons were used. Eplerenone $(50 \mu \mathrm{mol} / \mathrm{l})$ tested as a single modulator of arachidonate- or collagendependent aggregation did not exhibit any significant effect on platelet reactivity (data not shown).

\section{Discussion}

The present paper describes a pilot study whose aim is to verify whether aldosterone, cholesterol subfractions and Hcy, all considered as factors shaping the development of atherosclerosis (Toth 2005, Leiter 2000, Schaffer et al. 2014, Gromotowicz et al. 2011,
Borzyszkowska et al. 2012) are linked in CAD. It places a particular emphasis on aldosterone, the hormone, which may play the role of an upstream regulator of cholesterol and Hcy metabolism. The molecular association of total cholesterol and aldosterone seems to be quite obvious, since cholesterol plays the role of aldosterone precursor in the pathway of steroidogenesis (Williams et al. 2005). Hence, our present findings suggest that the studied patients are characterized by a higher expenditure of cholesterol necessary for aldosterone synthesis. Such a higher rate of cholesterol catabolism in the process of aldosterone production might potentially explain the increased concentrations of plasma aldosterone concomitant with lowered total and LDL cholesterol levels, highlighted by the presence of negative associations between these compounds measured in the peripheral blood. Indeed, in a hypoxic environment, characteristic of tissues supplied with blood by the atherosclerotic vasculature, aldosterone synthesis is enhanced in a steroidogenic acute regulatory protein (StAR)-dependent manner, as shown in pulmonary arterioles (Maron et al. 2014). The molecular explanation of cholesterol and aldosterone relationships in CAD cannot be precisely stated at the current state of knowledge, especially considering the fact that another group of researchers report a positive association between LDL cholesterol and plasma aldosterone in prehypertensive subjects, including young people (from 25 years old) (Syed and Quereshi 2012). This apparently contradicts our present results; it should be emphasized, however, that the group of patients enrolled in our study differed considerably with respect to a number of factors including age and hypertension. However, all deliberations on associations of aldosteronemia and lipidogram parameters in CAD may be strongly affected by the use of statins by investigated patients. The eventual interactions of aldosterone, lipidogram and statins should be further investigated.

Our findings show for the first time that Hcy, a potent cardiovascular toxicant (Mohan et al. 2008, Zhang et al. 2012, Lentz 2005, Hucks et al. 2004, Edirimanne et al. 2007), has been formed in adrenal glands stimulated by high concentrations of aldosterone following long-term in vitro incubation.

The concentrations of Hcy detected in our study in the incubation medium are quite low, and statistically significant changes were detected merely after longer incubation with "pathologically" high aldosterone concentrations. Regarding the aldosterone-induced changes 
in Hcy level taking place in the studied in vitro model, these fluctuations are significant and strong: the Hcy concentration noted after 12-h incubation with aldosterone was almost twice that observed in vehicletreated control. However, regarding whole body fluids, such an increase may appear as insignificant. Therefore, to extrapolate this phenomenon detected under in vitro conditions into in vivo circumstances, assuming that it is of physiological importance and may also occur in a living organism, it should be assumed that to effectively generate Hcy, large numbers of aldosterone molecules need to interact autocrinally with the tissues of the adrenal glands for a relatively long period of time. The most probable group of patients, who could potentially become exposed to such high aldosterone concentrations are those with a renal disease, who are undergoing hemodialysis (Krämer et al. 1990, Sjöberg et al. 2006). Importantly, in chronic kidney disease, Hcy is present at higher levels (Sjöberg et al. 2006, Shankar et al. 2008); therefore, plasma concentrations of aldosterone and Hcy may remain in some relationships in individuals with kidney disease and accompanying cardiovascular complications. In our experimental groups, the parameters informative for the possibly impaired kidney function (plasma levels of creatinine and cystatine C) revealed no differences between non-CAD and CAD groups and their values remained within the reference range. However, interestingly in both groups, plasma creatinine concentration positively correlated with plasma homocysteinemia, which stays in agreement with the opinions contributing the role in the regulation of plasma Hcy levels in the kidneys (Friedman et al. 2001, Potter et al. 2008). Importantly, plasma creatinine levels positively correlated with aldosteronemia only in CAD patients. As far as this preliminary study implicates the possibility that Hcy might be secreted by adrenal glands, it seems necessary to verify whether pathological aldosterone concentrations, even those much lower than $500 \mathrm{pg} / \mathrm{ml}$, could possibly trigger the synthesis of Hcy in the adrenal glands, i.e. acting as proatherogenic factor. However, based on the observation reported herein indicating that Hcy concentration declines following short-term stimulation of adrenal glands with higher concentrations of aldosterone, it can also be hypothesized that under some conditions aldosterone may be equally well considered an antiatherogenic acting hormone, i.e. lowering the synthesis of Hcy in the adrenal glands.

Nowadays, the molecular pathway of Hcy synthesis and transformation in the adrenal glands remains unknown. Our present outcomes suggest that aldosterone-mediated Hcy transformation in adrenal glands is not identical to the well-described transsulfuration occurring in kidneys (Friedman et al. 2001). In the case of Hcy transformation by classic thiol amino acid metabolic pathways, fluctuations in GSH and Cys concentrations should certainly accompany such reactions. However, such a presumption has not been confirmed in the in vitro part of the present study regarding that exposure of adrenal glands to aldosterone, where no changes in Cys, CysGly and GSH levels were observed. This observation is contrary to results from the ex vivo association analysis in CAD-positive subjects, in which Hcy level was found to be significantly positively associated with both cysteine and cysteinylglycine, but not with GSH (data not shown).

Our present study also shows that besides being associated with lipids and Hcy metabolism, aldosterone can also influence the progression of atherothrombosis through its direct action on blood platelets. It is believed that blood platelets are aldosterone-insensitive cells, since the ADP-stimulated platelet activation has remained unaffected by aldosterone (Moaraes et al. 2005); despite this, no report on arachidonic acid- or collagen-agonized platelets has been published. Aldosterone can augment platelet aggregation triggered by collagen and it significantly reduces aggregation induced by arachidonate. Interestingly, the preincubation of platelets with eplerenone, an antagonist of the mineralocorticoid receptor (MR), reverses the action of aldosterone on collagen-dependent platelet aggregation, but augments the antiplatelet action of aldosterone in the case of platelets aggregating in response to arachidonic acid. Therefore, this study presents the first confirmation that aldosterone has a dual, agonist-dependent action on platelet aggregation, and that the antihypertensive eplerenone offers additional benefits as an antiplatelet agent.

In summary, this paper describes a few new pathways of aldosterone involvement in the modulation of atherogenesis. First, aldosterone, specifically in CAD, associates negatively with some lipidogram parameters and positively with plasma homocysteinemia; the latter may potentially be derived from adrenal stimulation by high concentrations of aldosterone. Results suggesting any associations of aldosterone with atherogenic factors in patients with CAD should be carefully reevaluated, since the CAD group had received intense pharmacological treatment. Therefore, all the associations 
identified in this group could be potentially affected by drug metabolites. Second, aldosterone augments the proaggregatory action of collagen, but reduces platelet aggregation in response to arachidonate. Third, eplerenone, an antagonist of mineralocorticoid receptors, acts as an antiplatelet compound, respectively reducing or intensifying the action of aldosterone on collagen- or arachdionate-stimulated platelets.

\section{Conflict of Interest}

There is no conflict of interest.

\section{Acknowledgements}

This research was supported by the Medical University of Lodz (Lodz, Poland), No. 502-03/6-020 01/502-64-058, by the funds from the Polish Society of Metabolic Diseases (Lodz, Poland) and partially by the grant from the National Science Center (Cracow, Poland), No. UMO 2012/07/N/NZ1/03140.

\section{References}

AMANO T, MATSUBARA T, IZAWA H, TORIGOE M, YOSHIDA T, HAMAGUCHI Y, ISHII H, MIURA M, HAYASHI Y, OGAWA Y, MUROHARA T: Impact of plasma aldosterone levels for prediction of in-stent restenosis. Am J Cardiol 97: 785-788, 2006.

BALD E, GLOWACKI R: 2-chloro-1-methylquinolinium tetrafluoroborate as an effective and thiol specific UV-tagging reagent for liquid chromatography. J Liq Chromatogr Related Technol 24: 1323-1339, 2001.

BOOTH RE, JOHNSON JP, STOCKAND JD: Aldosterone. Adv Physiol Educ 26: 8-20, 2002.

BORZYSZKOWSKA J, STANISLAWSKA-SACHADYN A, WIRTWEIN M, SOBICZEWSKI W, CIECWIERZ D, TARGONSKI R, GRUCHALA M, RYNKIEWICZ A, LIMON J: Angiotensin converting enzyme gene polymorphism is associated with severity of coronary artery disease in men with high total cholesterol levels. J Appl Genet 53: 175-182, 2012.

EDIRIMANNE VE, WOO CW, SIOW YL, PIERCE GN, XIE JY: Homocysteine stimulates NADPH oxidase-mediated superoxide production lead endothelial dysfunction in rats. Can J Physiol Pharmacol 85: 1236-1247, 2007.

FRIEDMAN AN, BOSTOM AG, SELHUB J, LEVEY AS, ROSENBERG IH: The kidney and homocysteine metabolism. J Am Soc Nephrol 12: 2181-2189, 2001.

GROMOTOWICZ A, SZEMRAJ J, STANKIEWICZ A, ZAKRZESKA A, MANTUR M, JAROSZEWICZ E, ROGOWSKI F, CHABIELSKA E: Study of the mechanisms of aldosterone prothrombotic effect in rats. J Renin Angiotensin Aldosterone Syst 12: 430-439, 2011.

HILLAERT MA, LENTJES EG, KEMPERMAN H, VAN DER GRAAF, NATHOE HM, BEYGUI F, MONTALESCOT G, DOEVENDANS PA, WASSINK AM, VAN BELLE E: Aldosterone, atherosclerosis and vascular events in patients with stable coronary artery disease. Int J Cardiol 167: 1929-1935, 2013.

HUCKS D, THURAISINGHAM RC, RAFTERY MJ, YAQOOB MM: Homocysteine induced impairment of nitric oxide-dependent vasorelaxation is reversible by the superoxide dismutase mimetic TEMPOL. Nephrol Dial Transplant 19: 1999-2005, 2004.

IVANES F, SUSEN S, MOUQUET F, PIGNY P, CUILLERET F, SAUTIERE K, COLLET JP, BEYGUI F, HENNACHE B, ENNEZAT PV, JUTHIER F, RICHARD F, DALLONGEVILLE J, HILLAERT MA, DOEVENDANS PA, JUDE B, BERTRAND M, MONTALESCOT G, VAN BELLE E: Aldosterone, mortality, and acute ischaemic events in coronary artery disease patients outside the setting of acute myocardial infarction or heart failure. Eur Heart J 33: 191-202, 2012.

KAROLCZAK K, OLAS B: Mechanism of action of homocysteine and its thiolactone in hemostasis system. Physiol Res 58: 623-633, 2009.

KRÄMER BK, ULSHÖFER TM, MÜLLER GA, RESS KM, RISLER T: Regulation of plasma aldosterone during hemodialysis. Int J Artif Organs 13: 32-38, 1990.

LEITER LA: Low density lipoprotein cholesterol: is lower better? Can J Cardiol 16 (Suppl A): 20A-22A, 2000.

LENTZ SR: Mechanisms of homocysteine-induced atherothrombosis. Thromb Haemostasis 3: 1646-1654, 2005. 
MARON BA, OLDHAM WM, CHAN SY, VARGAS SO, ARONS E, ZHANG YY, LOSCALZO J, LEOPOLD JA: Upregulation of steroidogenic acute regulatory protein by hypoxia stimulates aldosterone synthesis in pulmonary artery endothelial cells to promote pulmonary vascular fibrosis. Circulation 130: 168-179, 2014.

MOHAN IV, JAGROOP IA, MIKHAILIDIS DP, STANSBY GP: Homocysteine activates platelets in vitro. Clin Appl Thromb Haemost 14: 8-18, 2008.

MORAES L, PAUL-CLARK MJ, RICKMAN A, FLOWER RJ, GOULDING NJ, PERRETTI M: Ligand-specific glucocorticoid receptor activation in human platelets. Blood 106: 4167-4175, 2005.

MUSANI SK, VASAN RS, BIDULESCU A, LIU J, XANTHAKIS V, SIMS M, GAWALAPU RK, SAMDARSHI TE, STEFFES M, TAYLOR HA, FOX ER: Aldosterone, C-reactive protein, and plasma B-type natriuretic peptide are associated with the development of metabolic syndrome and longitudinal changes in metabolic syndrome components: findings from the Jackson Heart Study. Diabetes Care 36: 3084-3092, 2013.

POTTER K, HANKEY GJ, GREEN DJ, EIKELBOOM JW, ARNOLDA LF: Homocysteine or renal impairment: which is the real cardiovascular risk factor? Arterioscler Thromb Vasc Biol 28: 1158-1164, 2008.

SCHAFFER A, VERDOIA M, CASSETTI E, MARINO P, SURYAPRANATA H, DE LUCA G; NOVARA ATHEROSCLEROSIS STUDY GROUP (NAS): Relationship between homocysteine and coronary artery disease. Results from a large prospective cohort study. Thromb Res 134: 288-293, 2014.

SHANKAR A, WANG JJ, CHUA B, ROCHTCHINA E, FLOOD V, MITCHELL P: Positive association between plasma homocysteine level and chronic kidney disease. Kidney Blood Press Res 31: 55-62, 2008.

SJÖBERG B, ANDERSTAM B, SULIMAN M, ALVESTRAND A: Plasma reduced homocysteine and other aminothiol concentrations in patients with CKD. Am J Kidney Dis 47: 60-71, 2006.

SYED SB, QURESHI MA: Association of aldosterone and cortisol with cardiovascular risk factors in prehypertension stage. Int J Hypertens 2012: 906327, 2012.

TOTH PP: High-density lipoprotein as a therapeutic target: clinical evidence and treatment strategies. Am J Cardiol 96: 50K-58K, 2005.

WILLIAMS GH: Aldosterone biosynthesis, regulation, and classical mechanism of action. Heart Fail Rev 10: 7-13, 2005.

ZHANG D, CHEN Y, XIE X, LIU J, WANG Q, KONG W, ZHU Y: Homocysteine activates vascular smooth muscle cells by DNA demethylation of platelet-derived growth factor in endothelial cells. J Mol Cell Cardiol 53: 487-496, 2012. 